\title{
A Distributed Online Service-Oriented Platform for the Airport Community
}

\author{
A. Mastoris, S. Mouzakitis, and D. Askounis
}

\begin{abstract}
Airports are expanding their operation from transportation centers to wide-ranging economic hubs. Despite the increased variety of the products and services that are been offered, the passenger's accessibility to products and services is still hindered. This paper presents a framework for the development of an airport infrastructure that offers out-of-the-box facilities for the design, administration and provision of services shared among the airport community, utilized by companies at the airport for its passengers.
\end{abstract}

Index Terms-Airport community, service delivery, service-oriented architectures.

\section{INTRODUCTION}

Airports are expanding their operation from transportation centers to wide-ranging economic hubs [1]. Since the early days of flying, the requirements for airports have increased in complexity and scale, as airports have evolved from pure transport hubs to Airport Cities that provide a growing number of services and facilities - not directly related to actual transport functions - such as duty free shops, restaurants, hotels and accommodation, banks and currency exchanges, etc [2], [3]. Despite the increased variety of the products and services that are been offered, the passenger's accessibility to products and services is still hindered, due to a number of factors, such as the limited time passengers spend in the airport and the wide shift in the passenger's demands, capabilities and means of communication within different areas the airport; for instance, the demands for services and relevant information in the duty free shopping area are radically different from the demands while in the boarding gates. On the other hand, the airport is equipped with multiple communication channels, both local (voice messages, audiovisual mediums, Bluetooth and wireless access points, internet kiosks, etc.) and location-independent (SMS, e-mails, www, etc.). These channels can be exploited to substantially accommodate easy and personalized access to information, products and services.

The discrete business units of an airport (airport community members) are in need of direct information "access" and online engagement of passengers / consumers. In order to achieve this, airports aim to provide a communication channel between two parties which will take into account the particularities of the Airport spaces and

Manuscript received November 1, 2012; revised January 23, 2013

Apostolis Mastoris is with the University of London, United Kingdom (email: apostolos.mastoris.2012@live.rhul.ac.uk)

Spiros Mouzakitis and Dimitris Askounis are with the National Technical University of Athens, Athens, Greece (email smouzakitis,askous@epu.ntua.gr, askous@epu.ntua.gr). facilities. One significant distinction of the airport from organizations of other business domains is the fact that the potential customers / passengers have limited time to discover and utilize the airport facilities and products. As such, the promotion of services and products needs to be meticulously planned and targeted in order to allow the efficient and quick discovery of passenger facilities and services. Moreover, another challenge is the differentiation of services and media depending on the location of the passenger at the airport. The provided services, as well as, the communication channels are different depending on whether the passengers are in the stores, in the boarding gates, security control checks, etc. Personalization of the offered services depending on the current location of the passenger was always a key issue for airports in both commercial and non-commercial services (departure notification service, priority lanes, lost and found services, directions). Therefore, there is an increasing demand for an airport infrastructure that supports the development and maintenance of innovative services through state-of-the-art communication channels and value added services, such as personalization, location-based identification and out-of-the-box development of airport-supported services. Academic and business studies have highlighted innovation through a service-oriented approach as the most important way to create value in the modern airport business environment [4]-[7].

Existing best business practices [6] suggest innovative airport services that focus on innovative technological applications. The revolution of the ICT domain in the past 5 years has drastically changed the way consumers interact with the service providers (social media channels, high level of personalization, viral marketing, etc). Nevertheless, these technological innovations have yet to achieve their maximum potential in terms of adoption level and use [8]. Despite the short-term relationship with the potential customers, the airport receives a vast volume of passengers, thus constituting an environment suitable for the creation of an Airport-wide community infrastructure that supports the implementation of fast-track, efficient services [9].

In this context, the present paper is oriented towards presenting a Community Airport platform framework (CAP), that aims to address the above mentioned challenges and opportunities. The following sections describe the proposed framework that has a pilot application [10] in Athens International Airport (AIA).

\section{A FRAMEWORK FOR ENABLING A COMMUNITY AIRPORT PLATFORM}

The main concept of the proposed framework is the 
development of an airport infrastructure that offers out-of-the-box facilities for the design, administration and provision of services shared among the airport community, utilized by companies at the airport for its passengers. The proposed framework aims to provide personalization capabilities for the travellers and to facilitate the availability and quality of services offered by the airport and enterprises operating in the airport ( See Fig. 1).

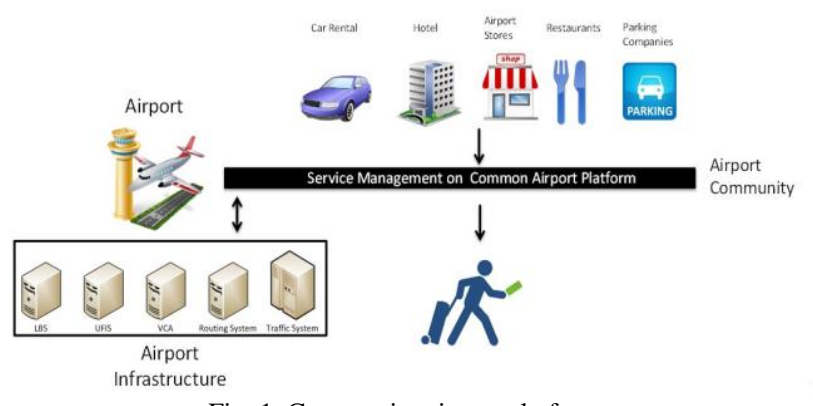

Fig. 1. Community airport platform.

The proposed framework can be applied for the development of an airport platform that offers user-friendly modules for the creation, provision and integration of services in the context of the airport environment. The framework allows the design and development of services from third-parties and facilitates its provision to members of the airport community (passengers, airport staff, visitors, enterprises, etc.). Moreover the framework provides content management capabilities, a personalization and a recommendation system. Lastly the framework contributes to the development of business models, processes and decision support mechanisms that will support the design and creation of a new airport service and thus it aims to significantly improve the competiveness of the enterprises operating in the airport as well as the quality of their services and products.

At its core, the CAP framework enables the interoperability of services coming from the various stakeholders of the Airport Community (stores, car rental companies, hotels, etc), modelled as an online airport services distribution platform. The CAP framework interoperates between these services while using common airport infrastructures (Airport TV screens -FIDS, IPTV, kiosks, Airport VCA system, UFIS flight data) and utilizing different communication channels (smartphones, internet, tables, laptops). All channels are interfaced with the CAP's platform content management system (CMS) to provide personalization of services based on the customer profile. The personalization system depends on the creation of profiles and the efficient operation through a recommender system. The user profiling is complemented by the current location / airport area of the customer.

Moreover the framework includes flexible business models where a variety of value-added airport related services are offered to the public quickly and automatically, within the short-term visit of customers to the airport community. For example, a car rental company announces a $30 \%$ discount offer for the next hour. By using an airport community platform that is based on the CAP framework, the car rental company creates a "discount" offer service that is been moderated by the Airport. The rental company has previously made an official agreement with the airport for access to the airport common infrastructure with different payment options (flat fee or revenue sharing) ( See Fig. 1).

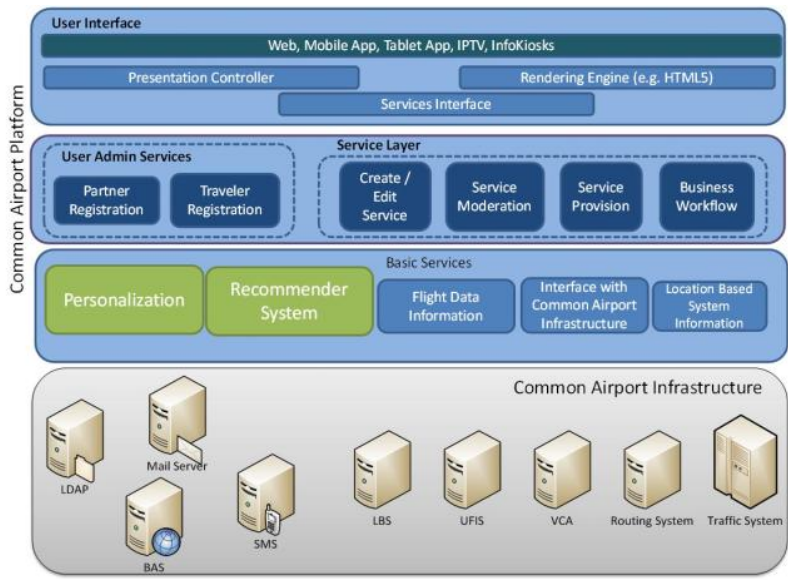

Fig. 2. Community airport platform elements.

Therefore the CAP framework consists mainly of the following components:

\section{A. Common Airport Platform}

This includes the operation of an online distributed platform for the intuitive creation, moderation and delivery of services (offers / discounts, ad-hoc advertisements, on-line purchases). The system will allow the creation of services by members of the airport community (car rental companies, airport hotels, parking owners, clothing shops, coffee/ bar / restaurant owners) and will facilitate the community members (airport passengers, workers, visitors, businesses, etc.). The platform is operated and maintained by the Airport which moderates all incoming requests for service creation.

The diversity of current software applications and data schemas raise a number of technical problems in the context of a centralized management of services coming from various clients, as well as coordination, operation and maintenance. The digital "map" of the airport includes a great number of complex applications and systems with the purpose of providing services that were created either by the Airport Company or by the Airport community partners and organizations. In this context the CAP framework identifies a common airport platform with the following components:

- Ad-hoc design and creation of online airport services system. This characteristic addresses the current absence of a consistent and "template"-based way to create a new service from companies operating within the area of the airport.

- Management and maintenance system: A central management and control system of the published services contributes to the quick, efficient and consistent service provision to consumers.

- Provision to passengers / consumers system: The system responsible for providing online services to consumers regardless of the type of service (e.g., informational, B2C / B2B transaction, etc.) or means of communication (e.g., airport infrastructures, audio messages, IPTV, smartphone, laptop, etc.).

The Common Airport Platform is an integrated platform for the creation and delivery of airport-related services that 
follow a service-oriented architecture. The platform provides utilities for the creation, promotion and exchange of information, products and services between the airport, members of the airport community and airport users, whether a simple visitor or passenger. The framework supports a unified system management and distribution of information, products and services through multiple communication systems.

Specifically, the main design elements of the proposed platform are:

- Mechanism for the creation, management \& distribution of services: The main goal is to design a mechanism for collecting, processing and publication of information services to all available communication channels and infrastructure of the airport.

- The automatic processing services: The main goal is to design a mechanism for the automatic processing of new services at the airport. This mechanism permits the design, submission for approval to the airport, and activation of a specific service.

- Targeted distribution of services: The main goal is to include a mechanism that allows the target identification and profiling, link of this profile to the corresponding services and according to the current location, the time frame in which the consumer visits the airport.

- Location identification system: This includes well know techniques and methods of identifying the current location inside the airport in order to provide appropriate services to them based on spatial parameters.

\section{B. Common Airport Infrastructure}

The airport has at his disposal a wide range of means and systems to share within the members of the airport community. Instead of creating specialized links with each individual member in order to provide access to these utilities, the airport can provide a centralized infrastructure which all members can use. In this model technical and business connections with its members are limited from $n^{*}(n-1)$ to $n$, thus significantly minimizing the administrative and operational costs, and improving the overall quality of the infrastructure provision.

Indicative systems that can be access through the Common Airport platform as a common infrastructure among all members of the airport community are:

- Flight Data offered by UFIS system (Arrivals, Departures, Flight Information)

- Current passenger's location through the airports LBS system,

- Information about the time and distance between current user location and the airport (Time2Airport)

- Information about regard to the time and distance between current user location and the boarding gate (Time2Gate)

- Airport VCA systems that provides data for the waiting queues at screening, security check points.

- Airport BAS system that includes data for the employees of the airport.

- On-the-spot information interfaces with passengers including info-kiosks, IPTVs, FIDS

\section{Personalization and Recommender System}

Personalization is a key aspect of the CAP framework. The personalization system produces a list of recommendations in two ways - through collaborative or content-based filtering. The Collaborative filtering approach builds a model from a traveller's past behavior (items previously purchased or selected and/or numerical ratings given to those items) as well as similar decisions made by other travellers, then use that model to predict items (or ratings for items) that the user may have an interest in. Content-based filtering approach utilizes a series of discrete characteristics of an airport service in order to recommend additional services with similar properties.

Personalization involves the process of adapting both the content and the means of communication to the passenger's liking. Administrative barriers is a significant factor to take into consideration (e.g. duty free travel are not allowed because the passenger is travelling within the country, or he/she is located at the gateway and is not allowed to go back). In general airport passengers can have two types of relationships with the airport, as a service provider: a) Long-term, where passengers are registered to the airport as clients in order to enjoy various privileges. All registered customers airport have membership card which will guarantee them special offerings. (B) Short-term where the customer will not register at the platform, but will have a temporary ticket (which is the boarding pass) so that all data related to this kind of passenger will be limited. In both types some profile characteristics will be dynamic. For example the history of transactions is updated with each new usage of a service or a purchase of a product and the current position of the passenger in the airport. In order to identify the location, both direct methods (smartphone, wifi signal) but also indirect (check-in status, boarding status).

The main element of the recommendation system is to model the characteristics of users and services and the development of techniques that correlate them. In order to create an adequate user profile, novel indirect information extraction occurs (e.g. identifying age level by habits as a consumer.

In addition to personalization of services, passengers' and services' profiles can be used to generate recommendations for users. The recommender system offers significant added value for the airport services, as the short-term visits of travelers is a barrier to its engagement. Short-term interaction with the passengers hinders their ability to discover products and services that interest them thus damaging both the providers and consumers. The CAP framework proposes a recommendation system that includes both characteristics of the products as well as the passengers' habits and history. The recommendations are created for products and services in real-time for customers that are within a close range. This boosts the dimension of the "duration" of the customer engagement. In order to exploit the user's visit lasts, the recommender system defines the concept of "session" on which the system will try to understand the trend in the consumer's behavior. E. g. The system can conclude whether the user is interested in cosmetics or beverages, or simply restaurants / food stores etc. The proposed system should update the data and the recommendation model in real time.

\section{Business Framework}

This component includes business models, workflows and decision-making mechanisms for the whole lifecycle of the 
CAP services (purchase, creation, maintenance, moderation, operation, and updates - expiration). Important aspect of the business framework is to support the automatic creation and processing of a new online service by identifying alternative business models, workflows and decision making needed to support the creation of new services.

A new service can be created and published by a company operating within the wide airport area that targets airport visitors and travellers. There are alternative business models based on which the service differentiate in terms of complexity, value creation and billing. Moreover the business workflow for the creation and operation of a new online service is required, such as service creation request procedures, service approval workflow from the airport and activation of the service workflow. Finally, the business framework identifies key performance indicators (KPI) and criteria to be taken into account to support decision making related to approval, activation and evaluation of a new service. Examples of such criteria include estimated revenue, level of compliance with safety and ethical standards, adoption and usage rate, etc.

The framework includes the following elements:

- The development of appropriate business models that can be used to provide new airport services.

- Identifying the workflow necessary to complete the cycle of creating a new service, the process of service requests moderation / approval by the airport based on the quality of the service and the terms of usage selection as well as identifying the billing model.

- A multi-criteria decision support system (based on the PROMETHEE method) for the realization of the above features.

\section{CONCLUSION}

The primary contribution of this paper was to present an overall framework for building a community airport platform that aims to distribute its information and infrastructure among the members of the airport community (rental companies, nearby hotels, airport stores and restaurants, airport employees, etc). The proposed framework includes the dynamic creation of airport-related services by the members of the airport community as well as its management in a unified manner by the Airport. The framework identifies essential technical and non-technical components for such an airport infrastructure.

The CAP platform can be used by companies operating within the airport zone in order improve their customer base and competitiveness, as well as the capabilities of the products and services they offer. The framework can be used as a design guide for Airports to build a common, service-oriented platform that acts as a service hub among the involved stakeholders. The integration of the framework will have the following advantages:

- Improve quality of service for travellers.

- Facilitate the development of new services by airport partners while taking advantage of the existing airport systems and data infrastructure as well as other value added services (personalization, recommendation system). The overall direct and management costs for the development of such services will be drastically reduced for both the airport partners and the airport, resulting in an increased economic activity within the airport zone.

- Better management and coordination with airports companies globally.

The framework, presented in this paper, can assist airports in identifying a common infrastructure to share among its partners and can serve as a guide for establishing an effective partnership strategy. In this way, this approach can contribute to achieving the full potential of the Airport user traffic and current infrastructures.

Future work includes on elaborating and validating the frameworks individual elements as well as reporting application results. Since December 2011, work is going forward on using the CAP framework in Athens International Airport, Greece, with four services (Discount tickets, Store Offers, Time to Gate Last minute Tickets,). Additional findings and results are expected during the system's pilot operation that will be circulated through further dissemination activities.

\section{ACKNOWLEDGMENT}

The project CAP is being co-financed by the European Regional Development Fund (ERDF) and national funds and is a part of the Operational Program «Competitiveness \& Entrepreneurship» (OPCE II), Measure "COOPERATION" (Action I).

\section{REFERENCES}

[1] R. L. Keast, D. C. Baker, and K. Brown, "Sustainable airport infrastructure: Balancing infrastructures for the airport metropolis," IGI Global, 2010.

[2] E. Fernandes and R. R. Pacheco, "Efficient use of airport capacity," Transportation Research Part A: Policy and Practice, vol. 36, no. 3, pp. 225-238, March 2002.

[3] J. Quartieri, M. Guida, C. Guarnaccia, S. D. Ambrosio, and D. Guadagnuolo, "Complex network applications to the infrastructure systems: The italian airport network case," WSEAS International Conference on Urban Planning and Transportation (UPT'07), Heraklion, Crete Island, Greece, July 22-24, 2008.

[4] A. Kapur, "Airport infrastructure: The emerging role of the private sector," World Bank, 1995.

[5] D. Starkie, The airport industry in a competitive environment: A United Kingdom perspective, Economics-Plus Ltd, London, United Kingdom.

[6] D. Jarach, "The evolution of airport management practices: Towards a multi-point, multi-service, marketing-driven firm," Journal of Air Transport Management, vol. 7, no 2, pp. 199-125, March 2001.

[7] R. Neufville and A. R. Odoni, Airport systems: Planning design, and management, McGraw-Hill Companies, 2003.

[8] T. Østerlie, O. M. Asak, O. G. Pettersen, and H. Tronhus, "Manufacturing accomplices: ICT use in securing the safety state at airport," IFIP Advances in Information and Communication Technology, vol. 318/2010, pp. 327-342, 2010.

[9] M. Martinez, R. Mercurio, and F. Rajola, "Governance models for the development of information systems in airport management companies," Editoriale Scientifica, 2010.

[10] Community Airport Platform Pilot Project. [Online]. Available: http://www.airpoint.gr

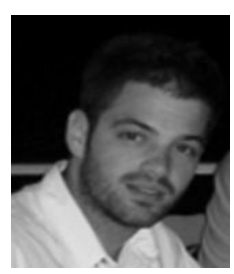

Apostolis Mastoris is a MSc student in information security at Royal Holloway, University of London. His current research is focused on Information Security, Algorithms, Web Applications and Distributed Systems. Apostolis Mastoris has worked as a researcher and software engineer in projects related to information security, management, analysis and design of Information Systems, ICT technologies, eGovernment and Open Data, and web development. 


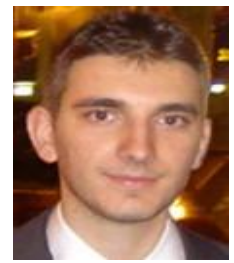

Spiros Mouzakitis is a research analyst for National Technical University of Athens (NTUA). He holds a diploma of Electrical and Computer Engineering from the National Technical University of Athens. He has nine years of industry experience in conception, analysis and implementation of information technology systems. His current research is focused on decision analysis in the field of eBusiness, enterprise interoperability and eGovernment. Spiros Mouzakitis has worked as a research analyst and software engineer in more than 15 projects related to the management, analysis and design of Information Systems, project management, market / impact analysis in the context of ICT technologies, Artificial Intelligence, web development and enterprise interoperability.

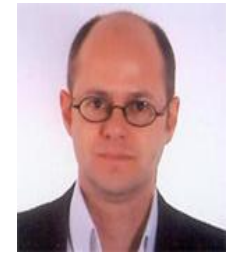

D. Askounis is an assistant professor in the School of Electrical and Computer Engineering of the National Technical University of Athens (NTUA). He has been involved in numerous research projects since 1988 in the areas of computer integrated manufacturing, enterprise resource planning, decision support, knowledge management, quality management business and data modeling and e-business. 